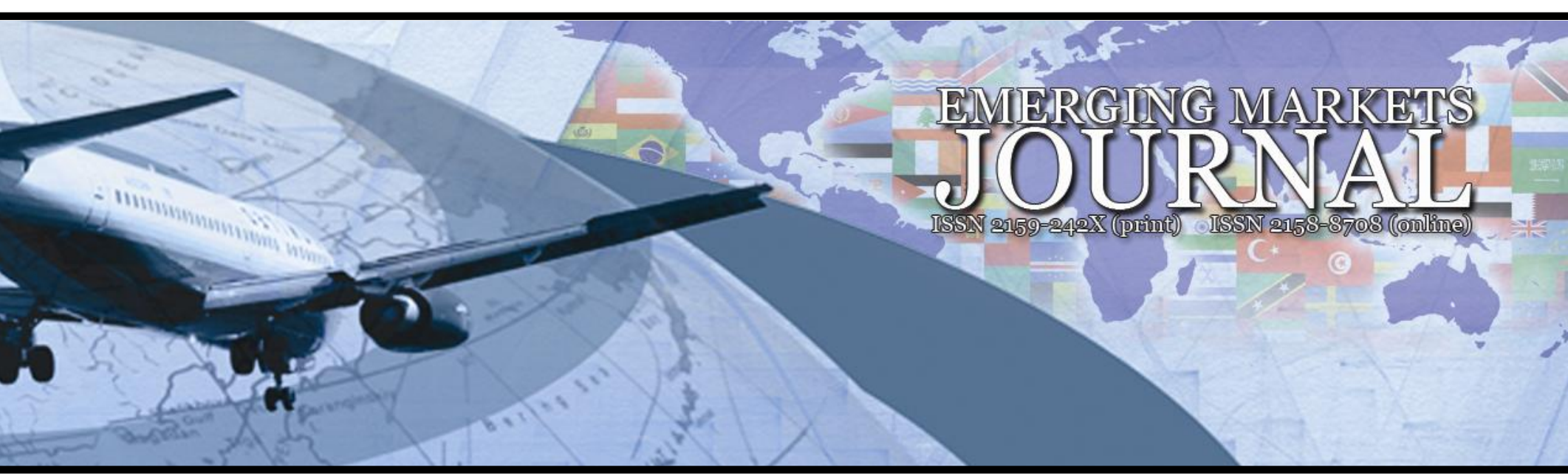

\title{
Heuristic Clustering Algorithms in Ad hoc Networks
}

\author{
Dr. Nevin Aydın
}

Artvin Çoruh University | e-mail: nevin.aydin@gmail.com

Volume 3 No 3 (2014) ｜ ISSN 2158-8708 (online) ｜ DOI 10.5195/emaj.2014.39 | http://emaj.pitt.edu |

\section{Abstract}

The clustering allows the geographical region to be covered into small zones in which each zone can be handled with a powerful node called clusterhead. The clusterheads have direct communication link with each of its members whereas the member nodes of a cluster must go through the clusterhead to communicate with each other. Since choosing clusterheads optimally is an NP-hard problem, existing solutions to this problem are based on heuristic (mostly greedy) approaches. In this paper, we present three well-known heuristic clustering algorithms: the Lowest-ID, the Highest-Degree, and the Node-Weight.

Keywords: clustering algorithms, clusterhead, heuristics, ad hoc networks

\section{(c) $)$ EY}

New articles in this journal are licensed under a Creative Commons Attribution 3.0 United States License.

\section{ULIS D-Sunt}

This journal is published by the University Library System of the University of Pittsburgh as part

of its $\mathrm{D}$-Scribe Digital Publishing Program, and is cosponsored by the University of Pittsburgh Press. 


\section{Heuristic Clustering Algorithms in Ad hoc Networks}

\author{
Dr. Nevin Aydin
}

\section{Introduction}

Wireless networks provide support for two types of network topologies: infrastructured and infrastructureless. The cellular networks, as an example for an infrastructured network, have emerged as the most widely used wireless networks that can support mobile users. The limitations of the infrastructure networks initiated the need for wireless networks without fixed infrastructures. Such networks are called mobile multihop radio networks, or ad hoc networks or peer-to-peer networks. These are in contrast to wireless networks that depend on a pre-existing fixed infrastructure of base stations (as in the case of cellular networks) (see Figure 1). In ad hoc networks, there is no such infrastructure and these networks are created dynamically on-the-fly in an ad hoc way. They play a critical role in places where a wired (central) backbone is neither available nor economical to build, such as law enforcement operations, battle field communications, disaster recovery situations, and so on. These situations demand a network where all the nodes including the base stations are potentially mobile, and communication must be supported untethered between any two nodes.
A multi-cluster, multi-hop packet radio network architecture for wireless systems should be able to dynamically adapt itself to the changing network configurations. Certain nodes, known as clusterheads, are responsible for the formation of clusters each consisting of a number of nodes (analogous to cells in a cellular network [6]. Clusterheads are also responsible for maintenance of the topology of the network. Unlike the base stations which are static entities, the clusterheads are mobile. Clusterheads are responsible for managing the nodes and doing the resource allocation to all the nodes within that cluster, just as a BS of a particular cell in a cellular network.

The remainder of the paper is organized as follows: Section 2 provides the concept of clustering in ad hoc networks while Section 3 discusses the heuristic clustering algorithms in detail. The paper is concluded in Section 4.

\section{Clustering}

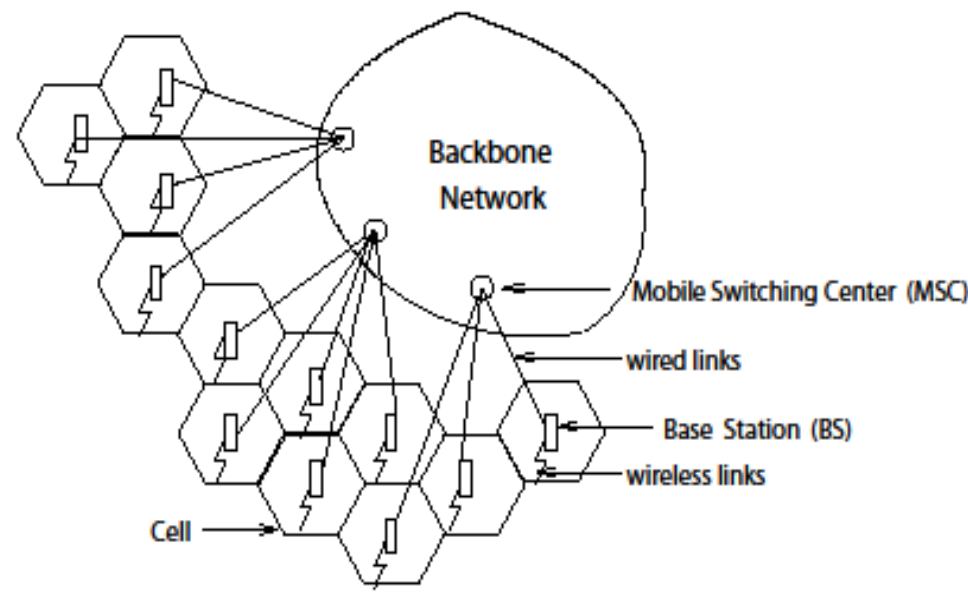

Figure 1. Cellular Network. 
The concept of dividing the geographical region to be covered into small zones has been presented implicitly as clustering in the literature [10]. Any node can become a clusterhead if it has the necessary functionality, such as processing and transmission power. Nodes register with the nearest clusterhead and become members of that cluster. Two nodes can communicate if they are within the transmission range of each other. These nodes are called neighbors of each other.

Clusters may change dynamically, reflecting the mobility of the underlying network. Due to the dynamic nature of the mobile nodes, their association and dissociation to and from clusters perturb the stability of the network and thus reconfiguration of clusterheads is unavoidable. This is an important issue since frequent clusterhead changes adversely affect the performance of other protocols such as scheduling, routing and resource allocation that rely on it. Since choosing clusterheads optimally is an NP-hard problem [3], existing solutions to this problem are based on heuristic (mostly greedy) approaches and none attempts to retain the stability of the network topology [3, 7]. We believe that a good clustering scheme should preserve the graph structure as much as possible when nodes are moving and/or the topology is slowly changing. Otherwise, re-computation of clusterheads and frequent information exchange among the participating nodes will result in high computation and communication overhead.

\section{Heuristic Clustering Algorithms}

There are several heuristics proposed for selecting clusterheads in ad hoc networks. The most well-known ones include: (1) the Highest-Degree heuristic, (2) the
Lowest-ID heuristic, and (3) the Node-Weight heuristic.

The Lowest-ID and the Highest-Degree were the two clustering algorithms that were based on the link-cluster architecture.

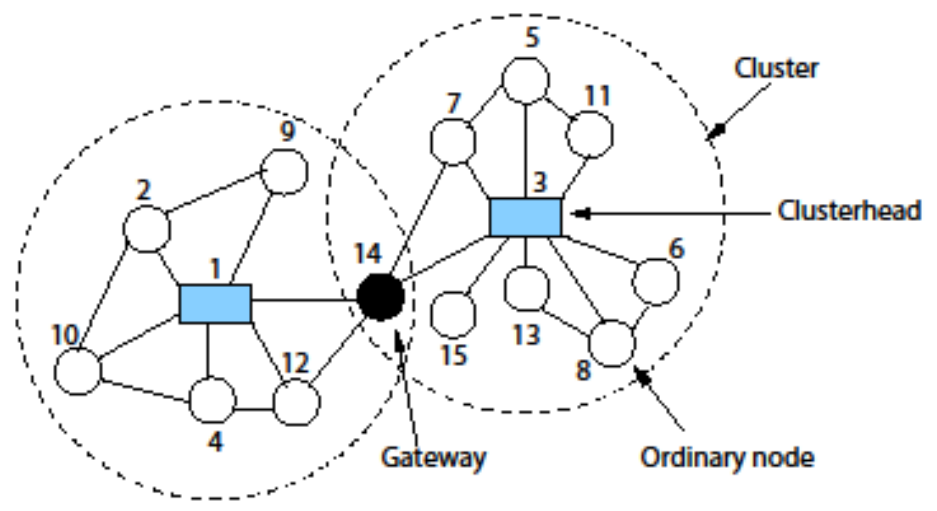

Figure 2. Lowest-ID and Highest Degree Heuristics

Example.

\section{III-1. The Lowest-ID Heuristic}

The Lowest-ID, also as known as identifierbased clustering, was originally proposed by Baker and Ephremides $[1,2,8]$. This heuristic assigns a unique ID to each node and chooses the node with the minimum ID as a clusterhead. Thus, the IDs of the neighbors of the clusterhead will be higher than that of the clusterhead. A node is called a gateway if it lies within the transmission range of two or more clusterheads. Gateway nodes are generally used for routing between clusters. Figure 2 shows nodes 1 and 3 as clusterheads since both hold the lowest node ID in their clusters and node 14 is a gateway node. 


\section{III-2. The Highest-Degree Heuristic}

The Highest-Degree, also known as connectivity-based clustering, was originally proposed by Gerla and Parekh $[9,11]$. Here the degree of a node is computed based on its distance from others. Each node broadcasts its ID to the nodes that are within its transmission range. A node $x$ is considered to be a neighbor of another node $y$ if $x$ lies within the transmission range of $y$. The node with maximum number of neighbors, that is, maximum degree is chosen as a clusterhead and any tie is broken by the unique node IDs. The neighbors of a clusterhead become members of that cluster and can no longer participate in the election process. Since no clusterheads are directly linked, only one clusterhead is allowed per cluster. Figure 2 shows a 15 node example, where nodes 1 and 3 are clusterheads since both have the highest number of neighbors.

\section{III-3. The Node-Weight Heuristic}

Basagni et al. $[4,5]$ proposed two algorithms, namely the distributed clustering algorithm (DCA) and the distributed mobility-adaptive clustering algorithm $(D M A C)$. In their approach, each node is assigned a weight (a real number $>=0$ ) based on its suitability of being a clusterhead. A node is chosen to be a clusterhead if its weight is highest among all of its neighbors; otherwise, it joins a neighboring clusterhead. The smaller node ID is chosen in case of a tie. The DCA makes an assumption that the network topology does not change during the execution of the algorithm. Thus, it is proven to be useful for "quasi-static" networks (when the nodes are either static or move very slowly). The DMAC algorithm, on the other hand, adapts itself to the network topology changes and can therefore be used for any mobile network

\section{Conclusions and Future Work}

In this paper, we have discussed the heuristic clustering algorithms in mobile ad hoc networks. Clustering is essentially means partitioning the network into different clusters and allows these clusters to communicate with each other in a wireless medium efficiently in order to achieve a connected network for data communication. Each cluster would be managed by a superior node called "clusterhead" and the election of these special nodes is similar to leader election algorithms in distributed systems. The heuristic algorithms discussed include the Highest-Degree heuristic, the Lowest-ID heuristic, and the Node-Weight heuristic.

\section{REFERENCES}

Baker, D.J. and Ephremides, A. (1981) “A Distributed Algorithm for Organizing Mobile Radio Telecommunication Networks," Proceedings of the Second International Conference on Distributed Computer Systems,"April, pp. 476-483.

Baker, D.J. and Ephremides, A. (1981), "The Architectural Organization of a Mobile Radio Network via a Distributed Algorithm," IEEE Transactions on Communications COM-29(11), November, pp. 1694-1701.

Basagni, S., Chlamtac, I., and Farago, A. (1997) “A Generalized Clustering Algorithm for Peer-to-Peer Networks," Proceedings of Workshop on Algorithmic Aspects of Communication (Satellite Workshop of ICALP). 
Basagni, S. (1999) "Distributed Clustering for Ad Hoc Networks," Proceedings of International Symposium on Parallel Architectures, Algorithms and Networks, June, pp. 310-315.

Basagni, S. (1999) “Distributed and MobilityAdaptive Clustering for Multimedia Support in MultiHop Wireless Networks," Proceedings of Vehicular Technology Conference, VTC 1999-Fall, Vol. 2, Amsterdam, September 19--22, pp. 889-893.

Black,U. (1999) Mobile and Wireless Networks, Prentice Hall.

Chlamtac, I. and Farago, A. (1999) “A New Approach to the Design and Analysis of Peer-to-Peer Mobile Networks," Wireless Networks, Vol. 5, No. 3, May, pp. 149-156.
Ephremides, A., Wieselthier, J.E., and Baker, D.J. (1987) "A design concept for reliable mobile radio networks with frequency hopping signaling," Proceedings of IEEE, Vol. 75, No. 1, January, pp. 56-73.

Gerla, M. and Tsai, J.T.C. (1995) "Multicluster, Mobile, Multimedia Radio Network," Wireless Networks, Vol. 1, No. 3, pp. 255-265.

Joa-Ng, M. and Lu, I.-T. (1999) “A Peer-to-Peer Zone-based Two-level Link State Routing for Mobile Ad Hoc Networks," IEEE Journal on Selected Areas in Communications, August, pp. 1415-1425.

Parekh, A.K. (1994) "Selecting Routers in Ad-Hoc Wireless Networks," In Proceedings of the SBT/IEEE International Telecommunications Symposium, August. 